\title{
Sentinel lymph node biopsy management after neoadjuvant treatment for breast cancer care
}

\author{
Giovanni Corso*,1, Alessandra Margherita De Scalzi ${ }^{1}$, Elisa Vicini ${ }^{1}$, Consuelo Morigi ${ }^{1}$, Paolo \\ Veronesi $i^{1,2}$ \& Viviana Galimberti ${ }^{1}$ \\ ${ }^{1}$ Division of Breast Cancer Surgery, European Institute of Oncology, via G. Ripamonti 435, 20141 Milano, Italy \\ ${ }^{2}$ Faculty of Medicine, University of Milan, Milano, Italy \\ *Author for correspondence: Tel.: +390294 375 161; giovanni.corso@ieo.it
}

"The SNB in node-positive patients with a radiologic axillary response after NAC is feasible and a sign of good practice.”

First draft submitted: 16 February 2018; Accepted for publication: 20 February 2018; Published online: 1 May 2018

Sentinel node biopsy (SNB) is part of a standard surgical practice and the 2017 National Comprehensive Cancer Network (NCCN) guidelines have recommended this procedure for most forms of breast cancer (BC), even in patients treated with neoadjuvant chemotherapy (NAC), although inflammatory tumors and cT4 cases are still excluded [1].

However, the correct indication for SNB after NAC treatment for BC is still controversial, particularly in patients with clinically involved axilla before NAC that shifts to clinically negative after treatment $(\mathrm{cN1}>\mathrm{cN} 0)$.

In the current issue, we will discuss this topic considering the latest literature data.

\section{Sentinel node biopsy}

The literature reported a significantly wide range of false-negative rates of SNB in patients with axillary lymph node metastasis before NAC, ranging from 10 to $30 \%$.

Therefore, we will consider data from the most prominent related studies.

\section{Retrospective studies}

- Takahashi: false-negative rate $24.5-27.3 \%$ (employed tracer ${ }^{99 \mathrm{~m}}$ Technetium) [2];

- Alvarado: false-negative rate 20.8\% (employed tracers: ${ }^{99 \mathrm{~m}}$ Technetium radiocolloid and blue dye) [3];

- Multicentric study from Mamounas: overall false-negative rate $14 \%$, but employing ${ }^{99 \mathrm{~m}}$ Technetium 5\% [4];

- Monocentric study from Hunt: false-negative rate 5.9\% [5].

Other studies have not reported a significantly high false-negative rate, indeed proving an excellent sentinel node (SN) detection rate, specifically the studies from Newman and from Canavese [6,7]. The first one evaluated 54 patients with metastatic axilla (confirmed by positive FNAC before neoadjuvant treatment); after primary treatment every patient underwent SNB and axillary clearance. The reported detection rate was $98 \%$ and $\mathrm{SN}$-negative cases had no residual tumor in the axilla. The second one considered 64 patients with a clinically positive axilla before neoadjuvant treatment; similarly, after primary treatment every patient underwent SNB and axillary clearance. The reported detection was high (93.8\%) and false-negative rate was quite low (8.7\%). An interesting consideration is the low false-negative rate reported by using ${ }^{99 \mathrm{~m}}$ Technetium.

\section{Prospective multicentric studies}

- European SENTinel NeoAdjuvant (SENTINA): false-negative rate 14.2\% (employed tracers: ${ }^{99 \mathrm{~m}}$ Technetium radiocolloid and blue dye) [8];

- The American College of Surgeons Oncology Group (ACOSOG) Z1071 trial: false-negative rate 12.6\%, 10\% if a minimum of three lymph nodes were excised (employed tracers: ${ }^{99 \mathrm{~m}}$ Technetium radiocolloid and blue dye).

Future $\because \cdots$ Medicine 
This study also underlines that when the metastatic node at the time of diagnosis was not resected the FNRs increased to $19 \%$ [9];

- The Canadian SNB Following Neoadjuvant Chemotherapy (SN FNAC) trial: FNRs 8.4\% [10].

Those recent trials have changed the surgical management of the axilla; in a study last year, the American surgeons incorporated this new data into their clinical practice [11].

The clinical significance of a high false-negative rate is still uncertain; it is not related to a complete pathological response and a high incidence of axillary relapses has not been reported even in studies reporting a false-negative rate $>10 \%$.

There is a significant variation between the reported detection rate and false-negative rate in literature. This wide range could be attributable to the individual expertise of the single centers and also to the structure of large retrospective studies without a standardized technique.

In order to minimize the false-negative rate and to optimize the detection rate, nodal status should be evaluated before and after neoadjuvant treatment with a clinical examination and an axillary ultrasound or with a positron emission tomography (PET). Particularly, if PET is negative after treatment, SNB at the time of the surgery is recommended, while axillary lymph node dissection is performed if PET results are positive after NAC [12].

Another technique described in the literature to minimize the FNRs at the lowest range 2-4\% [13] is combining the $\mathrm{SN}$ surgery with the resection of the metastatic node at the time of diagnosis. The metastatic node identified by a clip contained either blue dye, $99 \mathrm{mTc}$ activity or both in just $73 \%$ of the cases [14].

In order to reduce the false-negative rate $(<10 \%$ trial Z1071), a clip can be placed in the pathological node (FNAC positive) before NAC. In case of axillary downstaging ( $\mathrm{cN}+->\mathrm{cN} 0)$, the SNB could be performed [15]. The clipped node can be identified by ultrasound-guided wire localization or by a radioactive iodine seed, in order to verify the correspondence with the SNB and to resect the metastatic node at the time of diagnosis.

A study from the European Institute of Oncology in Milan considered 396 patients cT1-4 cN0/1/2 with negative axillary staging after neoadjuvant treatment who underwent SNB and axillary clearance only in the case of metastatic SN. Only one case of axillary relapse has been reported after a 61-month medium follow-up [16].

These preliminary results suggest that SNB is a safe procedure for cT1-T2 patients with negative axillary staging after neoadjuvant treatment, particularly in cases with complete pathological response on breast. SN status significant for prognosis and axillary clearance does not influence long-term survival.

Also, the 2017 San Gallen consensus conference confirmed SNB as the gold-standard procedure for patients with negative axillary staging $(\mathrm{cN} 1 / 2>\mathrm{cN} 0)$ after neoadjuvant treatment, while axillary clearance is still recommended with at least one metastatic node after NAC.

False-negative rate remains high if less than three lymph nodes are removed, if the metastatic node at the time of diagnosis was not resected and whether the panel was split regarding the indication to axillary clearance in case of residual micro-metastatic lymph node involvement.

\section{Axillary clearance}

The main indication for axillary clearance after neoadjuvant therapy is represented by cases with $\mathrm{cN} 1 / 2$ axillary staging (clinical examination or PET or axillary US) unchanged at the end of treatment. SN detection rate and false-positive rate should not be evaluated in this case because SNB should not be proposed. The patients with positive $\mathrm{SN}$ after neoadjuvant treatment should undergo axillary dissection.

\section{Preoperative lymphoscintigraphy}

A brief reference to this technique could be useful in the clinical practice. Preoperative lymphoscintigraphy after neoadjuvant treatment was debated in the past due to controversial data on detection rate and false-negative rate reported in the literature [17-20].

In this regard, we report two meta-analyses. The first one, performed by Xing et al., included 21 studies with a total of 1273 patients and demonstrated that with the use of a single radioactive tracer, SN detection rate can reach 90\% [21]. The second meta-analyses reported in the literature was performed by Kelly et al. [22], and 20 clinical trials with a total of $1799 \mathrm{BC}$ patients for which neoadjuvant treatment was indicated have been considered. These results confirmed a $90 \%(63-100 \%)$ detection rate after neoadjuvant treatment.

Another Italian study validates the lymphoscintigraphy after neoadjuvant treatment as a feasible technique with an $\mathrm{SN}$ detection rate of $96.9 \%$ [23]. 
From these two studies, we can then deduce that preoperative lymphoscintigraphy is a feasible procedure in patients who previously underwent neoadjuvant treatment.

\section{Conclusion}

The SNB in node-positive patients with a radiologic axillary response after NAC is feasible and a sign of good practice. Axillary clearance does not add any advantage to the patient; instead it is a possible source of complications.

\section{Financial \& competing interests disclosure}

The authors have no relevant affiliations or financial involvement with any organization or entity with a financial interest in or financial conflict with the subject matter or materials discussed in the manuscript. This includes employment, consultancies, honoraria, stock ownership or options, expert testimony, grants or patents received or pending, or royalties.

No writing assistance was utilized in the production of this manuscript.

\section{References}

1. Lyman GH. Appropriate role for sentinel node biopsy after neoadjuvant chemotherapy in patients with early-stage breast cancer. J. Clin. Oncol. 33(3), 232-234 (2015).

2. Takahashi M, Jinno H, Hayashida T, Sakata M, Asakura K, Kitagawa Y. Correlation between clinical nodal status and sentinel lymph node biopsy false negative rate after neoadjuvant chemotherapy. World J. Surg. 36(12), 2847-2852 (2012).

3. Alvarado R, Yi M, Le-Petross $\mathrm{H}$ et al. The role for sentinel lymph node dissection after neoadjuvant chemotherapy in patients who present with node-positive breast cancer. Ann. Surg. Oncol. 19(10), 3177-3184 (2012).

4. Mamounas EP, Brown A, Anderson S et al. Sentinel node biopsy after neoadjuvant chemotherapy in breast cancer: results from National Surgical Adjuvant Breast and Bowel Project Protocol B-27. J. Clin. Oncol. 23(12), 2694-2702 (2005).

5. Hunt KK, Yi M, Mittendorf EA et al. Sentinel lymph node surgery after neoadjuvant chemotherapy is accurate and reduces the need for axillary dissection in breast cancer patients. Ann. Surg. 250(4), 558-566 (2009).

6. Newman EA, Sabel MS, Nees AV et al. Sentinel lymph node biopsy performed after neoadjuvant chemotherapy is accurate in patients with documented node-positive breast cancer at presentation. Ann. Surg. Oncol. 14(10), 2946-2952 (2007).

7. Canavese G, Dozin B, Vecchio C et al. Accuracy of sentinel lymph node biopsy after neo-adjuvant chemotherapy in patients with locally advanced breast cancer and clinically positive axillary nodes. Eur. J. Surg. Oncol. 37(8), 688-694 (2011).

8. Kuehn T, Bauerfeind I, Fehm T et al. Sentinel lymph node biopsy in patients with breast cancer before and after neoadjuvant chemotherapy (SENTINA): a prospective, multicentre cohort study. Lancet Oncol. 14(7), 609-618 (2013).

9. Boughey JC, Suman VJ, Mittendorf EA et al. Sentinel lymph node surgery after neoadjuvant chemotherapy in patients with node-positive breast cancer: the ACOSOG Z1071 (Alliance) clinical trial. JAMA 310(14), 1455-1461 (2013).

10. Boileau JF, Poirier B, Basik M et al. Sentinel node biopsy after neoadjuvant chemotherapy in biopsy-proven node-positive breast cancer: the SN FNAC study. J. Clin. Oncol. 33(3), 258-264 (2015).

11. Caudle AS, Bedrosian I, Milton DR et al. Use of sentinel lymph node dissection after neoadjuvant chemotherapy in patients with node-positive breast cancer at diagnosis: practice patterns of American Society of Breast Surgeons members. Ann. Surg. Oncol. (10), 2925-2934 (2017).

12. Gilardi L, Paganelli G. Is a SUV cut-off necessary in the evaluation of the response of axillary lymph node metastases to neoadjuvant therapy? Eur. J. Nucl. Med. Mol. Imaging 37(11), 2202 (2010).

13. Caudle AS, Yang WT, Krishnamurthy $S$ et al. Improved axillary evaluation following neoadjuvant therapy for patients with node-positive breast cancer using selective evaluation of clipped nodes: implementation of targeted axillary dissection. J. Clin. Oncol. 34(10), 1072-1078 (2016).

14. Diego EJ, McAuliffe PF, Soran A et al. Axillary staging after neoadjuvant chemotherapy for breast cancer: a pilot study combining sentinel lymph node biopsy with radioactive seed localization of pretreatment positive axillary lymph nodes. Ann. Surg. Oncol. 23(5), 1549-1553 (2016).

15. Mittendorf EA, Caudle AS, Yang W et al. Implementation of the American College of Surgeons Oncology Group z1071 trial data in clinical practice: is there a way forward for sentinel lymph node dissection in clinically node-positive breast cancer patients treated with neoadjuvant chemotherapy? Ann. Surg. Oncol. 21(8), 2468-2473 (2014).

16. Galimberti V, Ribeiro Fontana SK, Maisonneuve P et al. Sentinel node biopsy after neoadjuvant treatment in breast cancer: five-year follow-up of patients with clinically node-negative or node-positive disease before treatment. Eur. J. Surg. Oncol. 42(3), 361-368 (2016).

17. Van Deurzen CH, Vriens BE, Tjan-Heijnen VC et al. Accuracy of sentinel node biopsy after neoadjuvant chemotherapy in breast cancer patients: a systematic review. Eur. J. Cancer 45(18), 3124-3130 (2009).

18. Dalus K, Reitsamer R, Holzmannhofer J et al. Lymphoscintigraphy in breast cancer patients after neoadjuvant chemotherapy. Diagnostic value and the work-up of sentinel node negative patients. Nuklearmedizin 50(1), 33-38 (2011). 
19. Ozmen V, Unal ES, Muslumanoglu ME et al. Axillary sentinel node biopsy after neoadjuvant chemotherapy. Eur. J. Surg. Oncol. 36(1), 23-29 (2010).

20. Lee S, Kim EY, Kang SH et al. Sentinel node identification rate, but not accuracy, is significantly decreased after preoperative chemotherapy in axillary node-positive breast cancer patients. Breast Cancer Res. Treat. 102(3), 283-288 (2007).

21. Xing Y, Foy M, Cox DD, Kuerer HM, Hunt KK, Cormier JN. Meta-analysis of sentinel lymph node biopsy after preoperative chemotherapy in patients with breast cancer. Br. J. Surg. 93(5), 539-546 (2006).

22. Kelly AM, Dwamena B, Cronin P, Carlos RC. Breast cancer sentinel node identification and classification after neoadjuvant chemotherapy - systematic review and meta analysis. Acad. Radiol. 16(5), 551-563 (2009).

23. Corso G, Grana CM, Gilardi L et al. Feasibility of lymphoscintigraphy for sentinel node identification after neo-adjuvant therapy. Ann. Ital. Chir. 88, 201-205 (2017). 